\title{
Higher rank Wilson loops from a matrix model
}

\author{
Sean A. Hartnoll ${ }^{a}$ and S. Prem Kumar ${ }^{b}$ \\ a DAMTP, Centre for Mathematical Sciences, \\ University of Cambridge, \\ Wilberforce Road, \\ Cambridge, CB3 0WA, UK. \\ ${ }^{b}$ Department of Physics, \\ University of Wales Swansea, \\ Singleton Park, \\ Swansea, SA2 8PP, UK. \\ E-mail: s.a.hartnoll@damtp.cam.ac.uk, s.p.kumar@swan.ac.uk
}

\begin{abstract}
We compute the circular Wilson loop of $\mathcal{N}=4$ SYM theory at large $\mathrm{N}$ in the rank $k$ symmetric and antisymmetric tensor representations. Using a quadratic Hermitian matrix model we obtain expressions for all values of the 't Hooft coupling. At large and small couplings we give explicit formulae and reproduce supergravity results from both D3 and D5 branes within a systematic framework.
\end{abstract}




\section{Background and motivation}

Wilson loops played a key role in the early development of the AdS/CFT correspondence [1]. The identification of the Wilson loop in $\mathcal{N}=4$ super Yang-Mills (SYM) theory with the dual fundamental string $[2,3]$ connected naturally with ideas of understanding the 'confining' strings of large $N$ gauge theory as fundamental strings [4]. At finite temperature, thermal Wilson loops enabled the identification of a deconfinement transition in the theory [5] and allowed the computation of the thermal interquark potential and screening length $[6-8]$.

There has recently been a renewed interest in Wilson loop operators in the AdS/CFT correspondence, with an underlying theme of understanding loops in higher representations of $S U(N)$. This has seen a convergence of various different research directions. Firstly, circular Wilson loops are half BPS operators and have been conjectured to be computed exactly, up to instanton corrections [9], to all orders in $N$ and $\lambda=g_{Y M}^{2} N$, from a quadratic Hermitian matrix model $[10,11]$. This matrix model makes nontrivial predictions for Wilson loops in higher representations which constitute a test of the AdS/CFT correspondence incorporating higher genus string effects [12].

Recently, the matrix model computation of a $k$ winding circular Wilson loop was reproduced using a D3 brane embedded in $A d S_{5}$ carrying $k$ units of worldvolume flux [13]. This beautiful match lead to further questions as it was not clear which rank $k$ representation of $S U(N)$ the D3 brane was computing. A complete dictionary was later proposed in [14] relating supersymmetric Wilson loops in an arbitrary representation to D3 and D5 branes in the dual geometry. According to this dictionary, a single D3 brane with $k$ units of flux is dual to a Wilson loop in the rank $k$ symmetric representation. It was therefore an open question to explain why the $k$ winding computation of [13], a priori certainly not the same as the rank $k$ symmetric respresentation, reproduced the supergravity result. This confusion lead us to the study of general symmetric representations in this paper.

A second line of interest has originated from the description of certain half BPS operators of $\mathcal{N}=4 \mathrm{SYM}$ theory carrying angular momentum as 'bubbling geometries' [15]. Wilson lines provide another family of half BPS operators and so it is natural to search for a bubbling geometry formalism describing the backreaction of the D3 and D5 branes, as well as a field theory understanding in terms of fermion droplets. Exciting progress in this direction is currently being made [16-18]. This line of interest inspired the succesful matching of a bulk D5 brane computation with a matrix model computation of the rank $k$ antisymmetric Wilson loop [19]. How- 
ever, that matrix model computation required an assumption that is not a priori justified and does not appear to indicate corrections to the supergravity result. This provided the motivation for us to do an honest matrix model computation of the antisymmetric representation in this paper.

Thirdly, computing the strong coupling thermal Wilson loops in higher representations is crucial for mapping out the phase structure of $\mathcal{N}=4$ SYM theory as a function of 't Hooft coupling and temperature [20]. It is an interesting open question whether there is a phase transition in the high temperature phase between weak and strong coupling physics, with implications for both black hole and plasma physics [21]. We recently initiated the required study of D3 and D5 branes in the AdS-Schwarzschild black hole background [22]. The results found for D5 branes in particular were curiously similar to those for the BPS circular spatial loop at zero temperature [19].

There have also been recent works on single Wilson loops in the fundamental representation $[23,24]$.

In this paper we study circular Wilson loops in rank $k$ symmetric and antisymmetric representations. Our computations will be in the large $N$ limit of the Hermitian matrix model of $[10,11]$. The expressions we derive will be exact in $\lambda$; we will take the large $\lambda$ limit only to obtain analytic results that may be compared with supergravity computations. A key formula in our framework is the contour integral representation of the symmetric and antisymmetric traces (2.26), which we then evaluate at large $N$ using a saddle point approximation. We go on to reproduce the known supergravity results for D3 and D5 branes $[13,19]$ as specific limits of this integral.

The results in this paper provide a check of the proposed holographic dictionary for general representations [14], and furthermore allows one to compute the corrections to the supergravity limit. This last fact clarifies the regime of validity of the supergravity computations. In the case of symmetric representations, we describe an interesting phenomenon whereby the nature of the saddle point changes qualitatively as a function of $k / N$ and $\lambda$. We finish by deriving an explicit formula for the Wilson loops at weak coupling.

Note: As we were typing up this work, the preprint [18] appeared which has some overlap with our results. 


\section{Circular Wilson loops}

The circular loop in the fundamental representation was first computed by Erickson, Semenoff and Zarembo [10] in perturbation theory by resumming a specific class of planar rainbow and ladder diagrams. It was further shown by these authors that cancellation of coordinate dependence in the planar Wick contractions makes it possible to map the result onto the solution of a large $N$ Hermitian matrix model with quadratic potential. Subsequently this result was argued by Drukker and Gross [11] to be the exact answer, up to nonperturbative instanton contributions [9], to all orders in $\lambda=g_{Y M}^{2} N$ and $1 / N$.

In this section we calculate BPS circular Wilson loops in the $k$-th rank antisymmetric and symmetric tensor representations of the $\mathrm{SU}(N) \mathcal{N}=4$ theory. Specifically, we are interested in the expectation value of the operator

$$
W_{\mathcal{R}}[U]=\frac{1}{\operatorname{dim}[\mathcal{R}]} \operatorname{Tr}_{\mathcal{R}}\left[\mathcal{P} \exp \left(i \oint_{C} d s\left(A_{\mu} \dot{x}^{\mu}+i \Phi_{I} \theta^{I}|\dot{x}|\right)\right)\right],
$$

where $\mathcal{R}$ denotes the representation and $\theta^{I}$ is a constant unit vector in $\mathbb{R}^{6}$. This choice breaks the $S O(6)$ R-symmetry of the $\mathcal{N}=4$ theory down to $S O(5)$. The dimensions of the rank $k$ symmetric and antisymmetric representions are respectively

$\operatorname{dim}\left[S_{k}\right]=\frac{(N+k-1) !}{k !(N-1) !}$ and $\operatorname{dim}\left[A_{k}\right]=\frac{N !}{k !(N-k) !}$. The contour $C$ will be chosen to be a spatial circle at zero temperature; this is then a BPS Wilson loop.

At finite temperature, the above operator with the contour $C$ chosen to be the Euclidean thermal circle yields the Maldacena-Polyakov loop. This is known to be an order parameter for the confinement/deconfinement transition in the strongly coupled field theory [5]. The Maldacena-Polyakov loop is not BPS in any natural sense since the finite temperature theory is not supersymmetric. Hence one does not expect the Maldacena-Polyakov loop to be related to the Hermitian matrix integral which computes zero temperature circular BPS Wilson loops. However, as mentioned in the introduction, the works of [22] and [19] suggest a tantalising connection. In particular, the operator (2.1) was computed in [22] using a probe D5 brane in the AdSSchwarzschild black hole background yielding the Maldacena-Polyakov loop for the finite temperature theory in the antisymmetric tensor representation. Remarkably, up to an overall temperature dependent normalisation factor, the result was found to be exactly the same as the zero temperature circular BPS Wilson loop in the antisymmetric tensor representation [19]. This suggests that at least in the supergravity $\left(\lambda=g_{Y M}^{2} N \gg 1\right)$ approximation there is a connection between Maldacena-Polyakov loops and Hermitian matrix models. This paper, however, will be concerned with the zero temperature theory and supersymmetric loops. 


\subsection{Circular Wilson loops from the matrix model}

The circular supersymmetric Wilson loop in the fundamental representation of $\mathrm{SU}(N)$ was calculated in [10] and [11] and shown to be computed by a Gaussian Hermitian matrix model. The latter is defined via the partition function

$$
\mathcal{Z}=\int[d M] \exp \left(-\frac{2 N}{\lambda} \operatorname{Tr}\left[M^{2}\right]\right)
$$

where $M$ is an $N \times N$ Hermitian matrix and $\lambda=g_{Y M}^{2} N$ is the 't Hooft coupling of the $\mathcal{N}=4$ gauge theory.

Assuming the conjectured relation between BPS Wilson loops and the matrix model for generic representations, circular Wilson loops in any representation $\mathcal{R}$ of the $S U(N)$ gauge theory are then given by

$$
\frac{1}{\operatorname{dim}[\mathcal{R}]}\left\langle\operatorname{Tr}_{\mathcal{R}}[U]\right\rangle=\frac{1}{\operatorname{dim}[\mathcal{R}]}\left\langle\operatorname{Tr}_{\mathcal{R}} e^{M}\right\rangle_{m m} .
$$

The expectation value on the right hand side is computed in the Gaussian matrix model above. This is a remarkable conjecture which we will employ to extract the Wilson loops in symmetric and antisymmetric tensor representations.

In an eigenvalue basis where $M=\operatorname{diag}\left\{m_{1}, m_{2}, m_{3}, \ldots, m_{N}\right\}$, the expectation values of the operators in the completely antisymmetric tensor representation can be expressed as

$$
\left\langle\operatorname{Tr}_{A_{k}}\left[e^{M}\right]\right\rangle_{m m}=\sum_{1 \leq i_{1}<i_{2}<\ldots<i_{k} \leq N}\left\langle\exp \left[m_{i_{1}}+m_{i_{2}}+\ldots+m_{i_{k}}\right]\right\rangle_{m m} \equiv\left\langle\alpha_{k}\right\rangle_{m m}
$$

which are simply $k$-th order symmetric polynomials $\alpha_{k}$ in the eigenvalues $\left\{e^{m_{i}}\right\}$. The $\left\{\alpha_{k}\right\}$ are encapsulated in the natural generating function for symmetric polynomials which is the spectral curve or characteristic polynomial associated to the matrix $e^{M}$

$$
F_{A}(t) \equiv \operatorname{det}\left(t+e^{M}\right)=\prod_{i=1}^{N}\left(t+e^{m_{i}}\right) .
$$

It is easily seen that $\alpha_{k}$ is the coefficient of $t^{N-k}$ in the spectral curve polynomial. It is most useful to express this relation as a contour integral in the complex $t$ plane

$$
\alpha_{k}=\frac{1}{2 \pi i} \oint d t \frac{F_{A}(t)}{t^{N-k+1}},
$$

At any finite $N$ the contour may be centred either around the origin or the point at infinity. Both definitions yield the same answer up to an overall sign. For infinite $N$ 
we will see that it is more convenient to think of the contour as encircling the point at infinity.

The $k$-th rank symmetric representations are given by the polynomials $\sigma_{k}$ in the eigenvalues $\left\{e^{m_{i}}\right\}$

$$
\left\langle\operatorname{Tr}_{S_{k}}\left[e^{M}\right]\right\rangle_{m m}=\sum_{1 \leq i_{1} \leq i_{2} \leq \ldots \leq i_{k} \leq N}\left\langle\exp \left[m_{i_{1}}+m_{i_{2}}+\ldots+m_{i_{k}}\right]\right\rangle_{m m} \equiv\left\langle\sigma_{k}\right\rangle_{m m}
$$

The generating functional for these polynomials is just the inverse of the characteristic polynomial ${ }^{1}$. We find it convenient to define it as

$$
F_{S}(t) \equiv \frac{1}{\operatorname{det}\left(1-t e^{M}\right)}=\prod_{i=1}^{N} \frac{1}{\left(1-t e^{m_{i}}\right)}
$$

As in the antisymmetric case, we can extract the polynomials $\sigma_{k}$ by a contour integral around the origin

$$
\sigma_{k}=\frac{1}{2 \pi i} \oint d t \frac{F_{S}(t)}{t^{k+1}}
$$

Note that the generating function $F_{S}(t)$ decays as $t^{-N}$ for large $|t|$, and has simple poles given by the positions of the eigenvalues of the matrix $e^{M}$, namely at $t=e^{-m_{i}}$. Near the origin $F_{S}(t) \sim 1+\sigma_{1} t+\sigma_{2} t^{2}+\ldots$, and hence the $\sigma_{k}$ are directly obtained from the above contour integral.

Our strategy will be to compute the expectation value of the generating functions in the matrix model

$$
\left\langle F_{A, S}(t)\right\rangle_{m m}=\frac{1}{\mathcal{Z}} \int \prod_{j=1}^{N}\left[d m_{j}\right] \Delta^{2}\left(m_{k}\right) F_{A, S}(t) \exp \left(-\frac{2 N}{\lambda} \sum_{i=1}^{N} m_{i}^{2}\right),
$$

where, as usual we have written the matrix integral in the eigenvalue basis at the expense of introducing a Jacobian factor, namely the Vandermonde determinant

$$
\Delta^{2}=\prod_{1 \leq i<j \leq N}\left(m_{i}-m_{j}\right)^{2}
$$

In principle it should be possible to evaluate the above integral for any finite $N$ and $\lambda$ by the method of orthogonal polynomials and extract the expectation values of BPS Wilson loops. However, in this paper we explore these expressions in the limit of large $N$ and fixed $\lambda$, which is relatively simpler and already very interesting.

\footnotetext{
${ }^{1}$ We would like to thank Asad Naqvi for pointing this out to us.
} 


\subsection{The large $N$ limit}

To take the large $N$ limit of the multi dimensional integral (2.10), we first rewrite it so as to make the $N$ dependence of various terms explicit

$$
\left\langle F_{A, S}(t)\right\rangle_{m m}=\frac{1}{\mathcal{Z}} \int \prod_{i=1}^{N}\left[d m_{i}\right] \exp \left(-S_{A, S}\left(\left\{m_{i}\right\}\right)\right),
$$

where

$$
S_{A, S}=\frac{2 N}{\lambda} \sum_{i=1}^{N} m_{i}^{2}-\sum_{i<j} \log \left(m_{i}-m_{j}\right)^{2}+\sum_{i=1}^{N}\left\{\begin{array}{c}
-\log \left(t+e^{m_{i}}\right) \text { for } S_{A} \\
+\log \left(1-t e^{m_{i}}\right) \text { for } S_{S}
\end{array}\right.
$$

Clearly the tree level quadratic term is of order $N^{2}$, as is the Vandermonde repulsive interaction between the eigenvalues. On the other hand the insertion of the characteristic polynomial term in the partition function only contributes at order $N$ in $S_{A, S}$. Therefore, in the large $N$ limit, the integral will be dominated by the usual Gaussian model saddle point configuration wherein the eigenvalues condense onto a cut in the complex eigenvalue plane with a spectral density characterised by the Wigner semi-circle distribution (see e.g. [25] and references therein)

$$
\rho(m)=\frac{2}{\pi \lambda} \sqrt{\lambda-m^{2}}, \quad-\sqrt{\lambda} \leq m \leq \sqrt{\lambda} .
$$

The spectral density is normalised so that

$$
\int_{-\sqrt{\lambda}}^{\sqrt{\lambda}} d m \rho(m)=1
$$

The large $N$ expectation values of the generating functionals are thus

$$
\begin{aligned}
\left\langle F_{A}(t)\right\rangle_{m m} & =\left\langle\exp \left(\operatorname{Tr} \log \left[t+e^{M}\right]\right)\right\rangle_{m m} \\
& \rightarrow \exp \left[N \int_{-\sqrt{\lambda}}^{\sqrt{\lambda}} d x \rho(x) \log \left(t+e^{x}\right)\right],
\end{aligned}
$$

and

$$
\begin{aligned}
\left\langle F_{S}(t)\right\rangle_{m m} & =\left\langle\exp \left(-\operatorname{Tr} \log \left[1-t e^{M}\right]\right)\right\rangle_{m m} \\
& \rightarrow \exp \left[-N \int_{-\sqrt{\lambda}}^{\sqrt{\lambda}} d x \rho(x) \log \left(1-t e^{x}\right)\right] .
\end{aligned}
$$

At this point we note that in the strict large $N$ limit there is a drastic change in the analytic structure of the characteristic polynomial $\left\langle F_{A}(t)\right\rangle_{m m}$. This can be 
understood in two different ways. Firstly, for any finite $N$ the representation of the polynomial as $\exp \left(\operatorname{Tr} \log \left(t+e^{M}\right)\right)$ yields only an $N$-th order polynomial upon a formal power series expansion around the origin. The coefficients of higher powers of $t$ cancel due to trace relations for large powers of the $N \times N$ matrix $e^{M}$. At infinite $N$, as is evident from (2.16), the power series in $t$ around the origin has infinitely many terms. This is due to the fact that the $N \rightarrow \infty$ limit has already been taken and one does not see traces involving powers of $e^{M}$ that are larger than $N$.

In addition, we note that on the real axis for $t<-e^{-\sqrt{\lambda}}$ the expression develops an imaginary part. This can be traced to a finite branch cut discontinuity along the negative real axis. The discrete zeros of the characteristic polynomial, which are the eigenvalues of $e^{M}$, coalesce into a continuum in the large $N$ limit producing the branch cut along $-e^{\sqrt{\lambda}} \leq t \leq-e^{-\sqrt{\lambda}}$. Hence at infinite $N$ our expression for the spectral curve does not yield a simple polynomial since it has a branch cut singularity in the $t$ plane along with a pole of order $N$ at infinity.

The generating function $\left\langle F_{S}(t)\right\rangle_{m m}$ for the symmetric Wilson loops also has a branch cut on the real axis for $e^{-\sqrt{\lambda}} \leq t \leq e^{\sqrt{\lambda}}$, which is due to the coalescing of $N$ simple poles in the large $N$ limit.

The large $N$ circular Wilson loop in the rank $k$ antisymmetric representation is then

$$
\frac{\left\langle\operatorname{Tr}_{A_{k}}[U]\right\rangle}{\operatorname{dim}\left[A_{k}\right]}=\frac{k !(N-k) !}{N !} \oint_{C} \frac{d t}{2 \pi i} t^{k-1} \exp \left[N \int_{-\sqrt{\lambda}}^{\sqrt{\lambda}} d x \rho(x) \log \left(1+e^{x} t^{-1}\right)\right],
$$

where we have absorbed the factor of $t^{N}$, see (2.6), into the exponent and the contour of integration $C$ is to be specified below. For the rank $k$ symmetric loops at large $N$, we have similarly

$$
\frac{\left\langle\operatorname{Tr}_{S_{k}}[U]\right\rangle}{\operatorname{dim}\left[S_{k}\right]}=\frac{k !(N-1) !}{(N+k-1) !} \oint_{C} \frac{d t}{2 \pi i} \frac{1}{t^{k+1}} \exp \left[-N \int_{-\sqrt{\lambda}}^{\sqrt{\lambda}} d x \rho(x) \log \left(1-e^{x} t\right)\right] .
$$

The problem of computing the Wilson loops is reduced to computing residues. This is quite straightforward in principle for finite $k=1,2, \ldots$ A more interesting question is whether it provides a useful way to explore the behaviour of these loops for generic values of $k$ that are comparable to $N$.

\subsection{Wilson loops at $k \sim N$}

A compact expression for the rank $k$ Wilson loops is obtained after a $t \rightarrow 1 / t$ variable 
change in (2.18) so that for the symmetric (S) and antisymmetric (A) loops we have

$$
\left\langle\operatorname{Tr}_{S_{k}, A_{k}}[U]\right\rangle=\frac{1}{2 \pi i} \oint_{C} d t \frac{1}{t^{k+1}} \exp \left[\mp N \int_{-\sqrt{\lambda}}^{\sqrt{\lambda}} d x \rho(x) \log \left(1 \mp e^{x} t\right)\right],
$$

where the - sign corresponds to the symmetric representation. The contour $C$ is chosen around $t=0$ and this encapsulates the prescription that the $k$-Wilson loops are the coefficients of positive powers of $t$ in the Laurent series around $t=0$. Indeed, this prescription works as we obtain

$$
\begin{gathered}
\left\langle\operatorname{Tr}_{S_{0}, A_{0}}[U]\right\rangle=1, \\
\left\langle\operatorname{Tr}_{S_{1}, A_{1}}[U]\right\rangle=N \int_{-\sqrt{\lambda}}^{\sqrt{\lambda}} d x \rho(x) e^{x}=\langle\operatorname{Tr}[U]\rangle, \\
\left\langle\operatorname{Tr}_{S_{2}, A_{2}}[U]\right\rangle=\frac{1}{2}\langle\operatorname{Tr} U\rangle^{2} \pm \frac{1}{2} N \int_{-\sqrt{\lambda}}^{\sqrt{\lambda}} d x \rho(x) e^{2 x}=\frac{1}{2}\left(\langle\operatorname{Tr} U\rangle^{2} \pm\left\langle\operatorname{Tr} U^{2}\right\rangle\right) .
\end{gathered}
$$

This is not surprising but provides a simple check of the prescription. Note that strictly speaking, the expressions (2.21) to (2.23) are not quite reliable within the large $N$ limit. We have kept terms which scale differently with $N$, while replacing $\left\langle(\operatorname{Tr} U)^{n}\right\rangle$ with $\langle\operatorname{Tr} U\rangle^{n}$ which is of course a consequence of the $N \rightarrow \infty$ limit. In any case, the direct approach of evaluating explicitly the residue at the origin is not useful for computing Wilson loops with $k$ comparable to $N$. To access these we need to evaluate the saddle point of the integrand at large $N$.

\subsection{Large $N$ saddle point}

The integral (2.20) yields the Wilson loops in the large $N$ theory and therefore only strictly makes sense in that limit. In the $N \rightarrow \infty$ limit we only need to consider the contribution from the saddle points of the integrand.

We are interested in the regime $k \rightarrow \infty$ with $k / N$ fixed. We introduce the fraction

$$
f=\frac{k}{N},
$$

and treat it as a continuous variable in the large $N$ limit. In addition, it is natural to perform the variable change

$$
t=e^{\sqrt{\lambda} z}
$$

which takes us from the complex plane to the cylinder. The points $t=0$ and $t=\infty$ are mapped to the points $z=\mp \infty$ respectively. 
For the antisymmetric case the branch cut singularity lying along the interval $\left(-e^{\sqrt{\lambda}},-e^{-\sqrt{\lambda}}\right)$ in the $t$ plane is mapped to the line joining $z=-1+i \frac{\pi}{\sqrt{\lambda}}$ and $z=1+i \frac{\pi}{\sqrt{\lambda}}$ and images thereof under translations by multiples of $\frac{2 \pi}{\sqrt{\lambda}} i$ in the $z$ plane. In the symmetric case the branch cut along $e^{-\sqrt{\lambda}}<t<e^{\sqrt{\lambda}}$ is mapped to the interval $-1<z<+1$ along the real axis and its images in the $z$ plane.

Using the explicit form of the Wigner semi-circle distribution and appropriately rescaling variables we find from $(2.20)$

$$
\left\langle\operatorname{Tr}_{S_{k}, A_{k}}[U]\right\rangle=\frac{\sqrt{\lambda}}{2 \pi i} \int_{C} d z \exp \left[\mp N\left(\frac{2}{\pi} \int_{-1}^{1} d x \sqrt{1-x^{2}} \log \left(1 \mp e^{-\sqrt{\lambda}(x-z)}\right) \pm f \sqrt{\lambda} z\right)\right] .
$$

The contour $C$ lies to the left of the branch cut, in the region $\operatorname{Re} z<-1$ and winds once around the $z$ plane cylinder.

In the $N \rightarrow \infty$ limit, we expect the integral to be dominated by the saddle points of the exponent. Hence we will first look for these. The saddle point equations are

$$
\frac{2}{\pi} \int_{-1}^{+1} d x \frac{\sqrt{1-x^{2}}}{1 \mp e^{\sqrt{\lambda}(x-z)}} \pm f=0 .
$$

The choice of upper signs yields the symmetric case. These equations will in general have solutions in the complex $z$ plane. Note that for certain values of $z$ we need to worry about potential divergences in the integrand in (2.27). The integral is then given by its Cauchy principal value along with an imaginary part. Such situations occur precisely when the solution lies on the branch cut and is not a stationary phase point. An explicit analysis of these equations is hard for generic $\lambda$. Instead we first study the limit of large $\lambda$ which can be directly compared with supergravity calculations.

\subsection{Supergravity limit}

The supergravity limit, $\lambda \rightarrow \infty$, leads to a drastic simplification of the exponent in (2.26) and the resulting saddle point equations (2.27). For the rest of this section, we will treat the antisymmetric and symmetric cases separately.

Antisymmetric: This corresponds to the equation (2.27) with the lower sign choice. Separating out the saddle point equation into real and imaginary pieces, we find that the imaginary part of the equation leads to the condition

$$
\sin (\sqrt{\lambda} \operatorname{Im} z)=0 .
$$


While this has the general solution $\sqrt{\lambda} \operatorname{Im} z=n \pi$, we will see below that only the solution $\operatorname{Im} z=0$ (and images) is picked out as the saddle point.

Now we turn to the real part of the saddle point equation. We first divide the $z$ plane into three regions: $\operatorname{Re} z<-1,-1 \leq \operatorname{Re} z \leq 1$ and $\operatorname{Re} z>1$. In each of these three regions the limit of large $\lambda$ leads to distinct asymptotic expressions. The saddle point equation can be expanded in powers of $e^{-\sqrt{\lambda}}$ in the large $\lambda$ regime to yield

$$
\begin{array}{r}
-f+O\left(e^{-\sqrt{\lambda}(|\operatorname{Re} z|-1)}\right)=0 \quad[\operatorname{Re} z<-1], \\
\frac{2}{\pi} \int_{-1}^{\operatorname{Re} z} d x \sqrt{1-x^{2}}-f+O\left(e^{-\sqrt{\lambda}(|\operatorname{Re} z|-1)}\right)=0 \quad[-1 \leq \operatorname{Re} z \leq 1],
\end{array}
$$

and finally

$$
(1-f)+O\left(e^{-\sqrt{\lambda}(|\operatorname{Re} z|-1)}\right)=0 \quad[\operatorname{Re} z>1] .
$$

It is clear that in the regions $\operatorname{Re} z>1$ and $\operatorname{Re} z<1$ there exist no consistent solutions in the strict $\lambda \rightarrow \infty$ limit for fixed $f$.

On the other hand, in the region $-1 \leq \operatorname{Re} z \leq 1$ we have a nontrivial equation that in general has solutions. Recalling that the branch cut extends between $z=$ $-1+i \frac{\pi}{\sqrt{\lambda}}$ and $z=1+i \frac{\pi}{\sqrt{\lambda}}$, we conclude that the stationary phase point lies at $\operatorname{Im} z=0$, on the real axis at

$$
z=\tilde{z} \in \mathbb{R}
$$

where $\tilde{z}$ satisfies

$$
\cos ^{-1} \tilde{z}-\tilde{z} \sqrt{1-\tilde{z}^{2}}=\pi(1-f),
$$

It is natural to rewrite this in an angular parametrisation

$$
\tilde{z}=\cos \theta_{k},
$$

where the subscript $k$ associates the solution to the rank $k$ representation. This gives

$$
\pi(1-f)=\left(\theta_{k}-\sin \theta_{k} \cos \theta_{k}\right) .
$$

Note that under $k \rightarrow N-k$, or equivalently $f \rightarrow 1-f$, the angular variable $\theta_{k}$ transforms to $\pi-\theta_{k}$. We will see in a moment that under this transformation the integral (2.26) remains invariant, reflecting the fact that antisymmetric representations of rank $k$ and $N-k$ are in fact identical.

The saddle point value of the integral is determined by the exponent in (2.26). Putting $z=\tilde{z}$ and taking the large $\lambda$ limit of (2.26) we obtain that the antisymmetric Wilson loop at large $k \sim N$ evaluates to

$$
W_{A_{k}}[U]=\frac{1}{\operatorname{dim}\left[A_{k}\right]}\left\langle\operatorname{Tr}_{A_{k}}[U]\right\rangle=\exp \left[\frac{2 N}{3 \pi} \sqrt{\lambda} \sin ^{3} \theta_{k}\right] \text {. }
$$


Here we have only kept the leading terms in the strict large $\lambda$ limit in the exponent. There are several noteworthy points about this result. The first is that it matches the answer obtained by a D5 probe brane calculation in $A d S_{5} \times S^{5}$ [19] and more surprisingly, the D5 brane probe calculation of the Polyakov loop in the AdS black hole background [22]. Although this answer was also obtained by a matrix model calculation in [19], the logic of that calculation is unclear ${ }^{2}$. In particular, our result will have corrections involving powers of $e^{-\sqrt{\lambda}}$ in the exponent. Such corrections were also present in the matrix model computation of [11] for the Wilson loop in the fundamental representation. Note also that the result is invariant under $\theta_{k} \rightarrow \pi-\theta_{k}$ reflecting the $k \rightarrow N-k$ symmetry for completely antisymmetric representations. Finally, as noted in [22] and [19], the above form of the action implies terms that appear nonanalytic from the point of view of the $1 / N$ expansion. Specifically, one obtains contributions involving powers of $\left(\frac{k}{N}\right)^{2 / 3}$ upon expanding the saddle point exponent in powers of $f=k / N$.

Symmetric: For the rank $k$ symmetric representation, the saddle point equation corresponds to choosing the upper signs in (2.27). This choice of sign completely changes the nature of solutions. First, by separating the equation into its real and imaginary parts we may show that there are no saddle points away from the real axis on the $z$ plane. Recall that in the symmetric case the branch cut is along the interval $-1<z<1$.

If we start with $\lambda \ll 1$, then we find a saddle point on the real $z$ axis with $\operatorname{Re} z<-1$. This saddle point can be treated analytically and we will do so in the following section. As we increase $\lambda$, however, we find that the saddle point moves in the positive direction along the real axis. At a critical value, $\lambda=\lambda_{c}$, the saddle point hits the branch point at $\operatorname{Re} z=-1$ and appears to move off into the second sheet. For a given $f$, from (2.27) we have that the critical point is reached when

$$
f=-\frac{2}{\pi} \int_{-1}^{1} \frac{d x \sqrt{1-x^{2}}}{1-e^{\sqrt{\lambda_{c}}(x+1)}} .
$$

Solving this equation we obtain a curve that splits the $(f, \lambda)$ plane into regions where a saddle point exists for negative real $z$ and where it does not. This curve is shown in figure 1.

\footnotetext{
${ }^{2}$ The calculation presented in [19] was in fact a computation of the cumulative distribution of the first $k$ eigenvalues in the cut using the large $N$ distribution $\frac{2}{\pi} \int_{-1}^{\cos \theta_{k}} d x x \sqrt{1-x^{2}}$. Although this matches the correct large $\lambda$ result, it differs from the correct finite $\lambda$ result as we already see from the $O\left(e^{-\sqrt{\lambda}}\right)$ corrections in our approach. Obtaining the correct answer using the approach of [19] involves computing the cumulative expectation value of $k$ distinct eigenvalues sprinkled randomly along the distribution governed by the Hermitian matrix model. These will give appropriate corrections to the result of [19]
} 


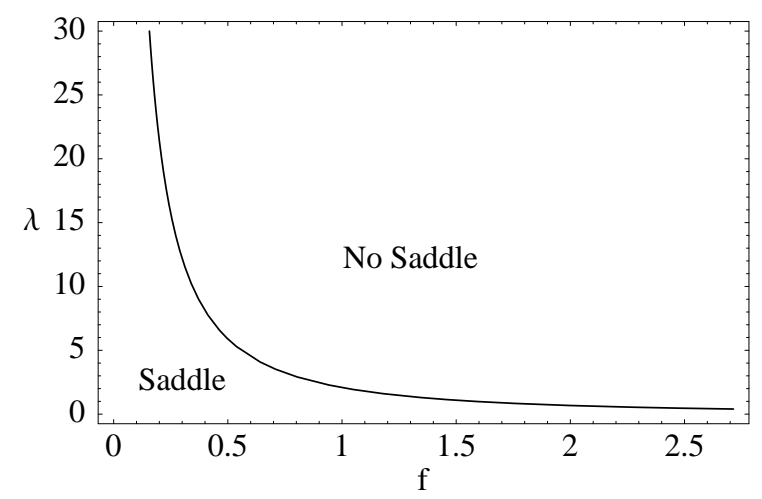

Figure 1: The existence of a saddle point at negative real $z$ depends on $f$ and $\lambda$ as shown.

The asymptotics of the curve in figure 1 may be derived analytically. We have

$$
\begin{aligned}
f \simeq \frac{2}{\lambda_{c}^{1 / 2}}+\cdots & \text { as } & \lambda_{c} \rightarrow 0, \\
f \simeq \sqrt{\frac{2}{\pi}} \frac{\zeta\left(\frac{3}{2}\right)}{\lambda_{c}^{3 / 4}}+\cdots & \text { as } & \lambda_{c} \rightarrow \infty .
\end{aligned}
$$

In the region where the saddle point exists, we can compute the location of the saddle point numerically and evaluate (2.26) at the saddle, thereby giving us the Wilson loop expectation value. However, at any fixed $f$, once the coupling is sufficiently strong there are no saddle points on the first sheet of the complex plane. We are aiming for analytic expressions in the $\lambda \rightarrow \infty$ limit. Since it is not clear how to pick up saddle point contributions from the second Riemann sheet, we will adopt a different methodology to evaluate (2.26) for the symmetric representation.

The integral has no singularitites at $\operatorname{Re} z \rightarrow+\infty$ (corresponding to $t \rightarrow \infty$ in our initial coordinates). Its only singularity is the branch cut on the real axis. We may therefore deform the contour to wrap the branch cut so that the integral reduces to the discontinuity across the cut

$$
\begin{array}{r}
\left\langle\operatorname{Tr}_{S_{k}}[U]\right\rangle=\frac{\sqrt{\lambda}}{\pi} \operatorname{Im} \int_{-1}^{1} d y \exp \left[-N\left(\frac{2}{\pi} \int_{-1}^{y} d x \sqrt{1-x^{2}} \log \left(e^{\sqrt{\lambda} y}-e^{\sqrt{\lambda} x}\right)+\right.\right. \\
\left.\left.+\frac{2}{\pi} \int_{y}^{1} d x \sqrt{1-x^{2}} \log \left(e^{\sqrt{\lambda} x}-e^{\sqrt{\lambda} y}\right)+2 i \int_{-1}^{y} d x \sqrt{1-x^{2}}+f \sqrt{\lambda} y\right)\right] .
\end{array}
$$

The Wilson loop is thus given by a definite integral over the real variable $y$ and we can unambiguously evaluate it using the stationary phase method at large $N$, by looking for the saddle points of the integrand analytically continued to the complex plane. 
We find two sets of saddle points in the large $\lambda$ limit, one whose location depends only on the combination $f \sqrt{\lambda}$ in the strong coupling limit, and another set whose location is independent of $\lambda$ in this limit.

The first of these saddle points occurs along the negative real axis and solves the equation

$$
\frac{2}{\pi} \sqrt{\lambda} \int_{-1}^{+1} \frac{d x \sqrt{1-x^{2}} e^{\sqrt{\lambda} y}}{e^{\sqrt{\lambda} y}-e^{\sqrt{\lambda} x}}+4 i \sqrt{1-y^{2}}+f \sqrt{\lambda}=0 .
$$

The factor of 4 in this expression arises for the following reason. Analytically continuing the exponent of (2.39) into the complex $y$ plane and in particular to large negative values of $y$ yields an extra imaginary contribution from the logarithm which then doubles the coefficient of the third integral in the exponent.

At large $\lambda$ and a fixed value of $\kappa$, where

$$
\kappa=\frac{f \sqrt{\lambda}}{4}
$$

we find a solution to the saddle point equation (2.40) along the negative real axis at

$$
y=y_{1}=-\sqrt{1+\kappa^{2}}+O\left(e^{-\sqrt{\lambda}\left(\left|y_{1}\right|-1\right)}\right) .
$$

Plugging this back into (2.39) we obtain the contribution to the Wilson loop from this saddle point as

$$
W_{1}=\exp \left[2 N\left(\kappa \sqrt{1+\kappa^{2}}+\sinh ^{-1} \kappa\right)\right],
$$

which is exactly the result found by Drukker and Fiol [13] using a probe D3 brane in $A d S_{5} \times S^{5}$. We have only kept the leading terms in the large $N$, large $\lambda$ limit in the exponent. In particular we have ignored phases. This is only one of the two contributions to the symmetric Wilson loop.

The integrand (2.39) appears to have yet another saddle point at large $\lambda$ in the complex plane, whose position is independent of $\lambda$ in this limit. To see this we directly take the large $\lambda$ limit of the exponent (2.39). Once we have taken this limit we can look for the saddle point. The condition turns out to be very similar to (2.30), obtained earlier in the antisymmetric case

$$
\frac{2}{\pi} \int_{-1}^{y} d x \sqrt{1-x^{2}}+f=0
$$

except that $y$ can be anywhere in the complex plane. It is obvious that there are no real solutions to this equation. This is a key difference between the symmetric and antisymmetric representations. The expressions obtained are only superficially 
similar. It may be checked numerically that the equation has solutions in the complex plane. In terms of a complex angular variable, the complex saddle point in the $y$ plane

$$
y_{2}=\cos \phi_{k} \in \mathbb{C},
$$

is determined by

$$
(1+f) \pi=\phi_{k}-\sin \phi_{k} \cos \phi_{k} .
$$

This does not have any solutions for real $\phi_{k}$ between 0 and $\pi$. The main difference from the antisymmetric case (2.35) is that now the left hand side is greater than unity.

This solution contributes to the symmetric Wilson loop in the limit of large $\lambda$ as

$$
W_{2}=\exp \left[-\frac{2 N}{3 \pi} \sqrt{\lambda} \operatorname{Re}\left(\sin ^{3} \phi_{k}\right)\right],
$$

where we have ignored phases and terms that are subleading in the exponent in the large $\lambda$ regime. We have checked numerically that for generic values of $\lambda$ and $f$ the exponent turns out to be positive at the saddle point and hence is nonzero in the large $N$ limit.

The Wilson loop in the symmetric representation is given by the sum of these two stationary point values

$$
W_{S_{k}}[U]=\frac{1}{\operatorname{dim}\left[S_{k}\right]}\left\langle\operatorname{Tr}_{S_{k}}[U]\right\rangle=W_{1}+W_{2} .
$$

Depending on the specific values of $f=k / N$ and $\lambda$, one of the two stationary points provides the answer in the large $N$ theory. The limit that is relevant for direct comparison with the D3 brane computation in supergravity is $\lambda \rightarrow \infty$ with $\kappa$ fixed. In this limit the saddle point $W_{1}$ is dominant and therefore our computations match the D3 brane result of [13]. Another limit in which $W_{1}$ dominates is the large $\lambda$ limit with $k / N$ fixed, since its exponent scales as $2 N \kappa^{2} \sim N \lambda$ while the exponent of $W_{2}$ only scales as $N \sqrt{\lambda}$. For general values of $\lambda$ and $f$, either saddle could dominate.

\subsection{Perturbative limit}

Interesting explicit results may also be obtained in the opposite limit, $\lambda \rightarrow 0$. In this regime, a unified treatment of the symmetric and antisymmetric cases is possible.

The first step is to solve the saddle point condition (2.27) perturbatively in $\lambda$. As in the previous subsection, the saddle point must lie on the real axis: $z=\tilde{z} \in \mathbb{R}$. 
One finds the following expansion to the lowest two orders

$$
e^{-\sqrt{\lambda} \tilde{z}}=\left( \pm 1+\frac{1}{f}\right)+\frac{\lambda}{8}\left( \pm 3+\frac{1+2 f^{2}}{f}\right)+\cdots
$$

As elsewhere, the upper choice of signs corresponds to the symmetric case.

Substituting the expansion (2.49) into (2.26) and expanding the expression in the exponent, we obtain the following result for the perturbative Wilson loop to leading order as $\lambda \rightarrow 0$

$$
W_{S_{k}, A_{k}}[U]=\exp \left[\frac{N \lambda}{8}\left(f \pm f^{2}\right)\right] .
$$

We can recognise the exponent of this result as the quadratic Casimir of the symmetric and antisymmetric representations of $S U(N)$ in the large $N$ limit with $f=k / N$ kept fixed. This observation strongly suggests the following perturbative result for a general representation

$$
W_{\mathcal{R}}[U]=\exp \left[\frac{\lambda C_{2}(\mathcal{R})}{8 N}\right] .
$$

The appearance of the quadratic Casimir is not surprising and the linear dependence on $\lambda$ is consistent with a one-loop perturbative gauge theory result. The coefficient is a nontrivial result of the $\mathcal{N}=4$ gauge theory.

A more detailed study at weak coupling in the symmetric case provides a pleasing consistency check of the strong coupling results. There exists a saddle point which is the weak coupling counterpart of the saddle (2.42) that we matched with the supergravity D3 brane result. This may be seen most easily in the limit $\lambda \rightarrow 0$ with fixed $\kappa \gg 1$. In this case the saddle point equation (2.40) is solved by

$$
y=y_{1}=-\sqrt{1+\kappa^{2}}+\mathcal{O}\left(\kappa^{-1}\right) .
$$

Evaluating the Wilson loop on this saddle point yields precisely the large $\kappa$ limit of the result (2.43). Thus although we might appear to be far from the supergravity regime, we can still make contact with the D3 brane result of [13].

\section{Conclusions}

In this paper we have used the quadratic Hermitian matrix model of $[10,11]$ to compute circular higher rank Wilson loops in $S U(N) \mathcal{N}=4$ SYM theory. In particular, we gave an expression for the rank $k$ symmetric and antisymmetric representations in terms of a single contour integral. This integral expression is valid for all values of the 't Hooft coupling and at large $N$ may be computed in the saddle point approximation. 
Taking the strong coupling limit, $\lambda \rightarrow \infty$, we reproduced analytically both the supergravity D3 brane result of Drukker and Fiol [13], using the symmetric representations, and the supergravity D5 brane result of Yamaguchi [19], using the antisymmetric representations. This provides nontrivial evidence for the Wilson loop dictionary proposed in [14] and of the AdS/CFT correspondence more generally: the match involves higher genus terms, as $k / N$ was kept fixed in the large $N$ limit. Our results also clear up confusions in the literature, in which the D3 brane result was initially matched with a multiply wound loop while the D5 brane result was matched with a matrix model computation whose validity was not a priori obvious.

Away from the strong coupling limit we find corrections to the supergravity

results governed by $e^{-\sqrt{\lambda}}$. These corrections are computable, if unilluminating at present, within our framework. We also computed the Wilson loops in the small $\lambda$ limit, and were led to a simple formula that we expect to be true for an arbitrary tensor representation with mixed symmetries.

The interpolation from weak to strong coupling appears to be uneventful in the antisymmetric case. In contrast, there is a very interesting phenomenon in the symmetric case. At a critical value of the coupling, the saddle point of the contour integral hits a branch point and moves off the real axis and into a second Riemann sheet. We have shown the dependence of the critical coupling on $k / N$, but it seems likely that there is interesting physics around this point still to be uncovered.

The formalism we have presented can also be used at finite $N$, with the matrix model expectation values evaluated using orthogonal polynomials rather than contour integrals. This may be another interesting direction for future work.

\section{Acknowledgements}

We are particularly grateful to Asad Naqvi for numerous helpful comments in the course of this work. We would also like to thank Adi Armoni, Tim Hollowood and Harald Ita. SAH is supported by a research fellowship from Clare college, Cambridge. SPK is supported by a PPARC advanced fellowship.

\section{References}

[1] J. M. Maldacena, "The large N limit of superconformal field theories and supergravity," Adv. Theor. Math. Phys. 2 (1998) 231 [Int. J. Theor. Phys. 38 (1999) 
1113] [arXiv:hep-th/9711200].

[2] J. M. Maldacena, "Wilson loops in large N field theories," Phys. Rev. Lett. 80 (1998) 4859 [arXiv:hep-th/9803002].

[3] S. J. Rey and J. T. Yee, "Macroscopic strings as heavy quarks in large N gauge theory and anti-de Sitter supergravity," Eur. Phys. J. C 22 (2001) 379 [arXiv:hep-th/9803001].

[4] A. M. Polyakov, "String theory and quark confinement," Nucl. Phys. Proc. Suppl. 68 (1998) 1 [arXiv:hep-th/9711002].

[5] E. Witten, "Anti-de Sitter space, thermal phase transition, and confinement in gauge theories," Adv. Theor. Math. Phys. 2 (1998) 505 [arXiv:hep-th/9803131].

[6] S. J. Rey, S. Theisen and J. T. Yee, "Wilson-Polyakov loop at finite temperature in large N gauge theory and anti-de Sitter supergravity," Nucl. Phys. B 527 (1998) 171 [arXiv:hep-th/9803135].

[7] A. Brandhuber, N. Itzhaki, J. Sonnenschein and S. Yankielowicz, "Wilson loops in the large N limit at finite temperature," Phys. Lett. B 434 (1998) 36 [arXiv:hep-th/9803137].

[8] K. Landsteiner and E. Lopez, "Probing the strong coupling limit of large N SYM on curved backgrounds," JHEP 9909, 006 (1999) [arXiv:hep-th/9908010].

[9] M. Bianchi, M. B. Green and S. Kovacs, "Instanton corrections to circular Wilson loops in N = 4 supersymmetric Yang-Mills," JHEP 0204, 040 (2002) [arXiv:hep-th/0202003].

[10] J. K. Erickson, G. W. Semenoff and K. Zarembo, "Wilson loops in N = 4 supersymmetric Yang-Mills theory," Nucl. Phys. B 582, 155 (2000) [arXiv:hep-th/0003055].

[11] N. Drukker and D. J. Gross, "An exact prediction of N $=4$ SUSYM theory for string theory," J. Math. Phys. 42, 2896 (2001) [arXiv:hep-th/0010274].

[12] D. J. Gross and H. Ooguri, "Aspects of large N gauge theory dynamics as seen by string theory," Phys. Rev. D 58, 106002 (1998) [arXiv:hep-th/9805129].

[13] N. Drukker and B. Fiol, "All-genus calculation of Wilson loops using D-branes," JHEP 0502, 010 (2005) [arXiv:hep-th/0501109].

[14] J. Gomis and F. Passerini, "Holographic Wilson loops," arXiv:hep-th/0604007.

[15] H. Lin, O. Lunin and J. Maldacena, "Bubbling AdS space and 1/2 BPS geometries," JHEP 0410 (2004) 025 [arXiv:hep-th/0409174].

[16] S. Yamaguchi, "Bubbling geometries for half BPS Wilson lines," arXiv:hep-th/0601089. 
[17] O. Lunin, "On gravitational description of Wilson lines," arXiv:hep-th/0604133.

[18] K. Okuyama and G. W. Semenoff, "Wilson Loops in N=4 SYM and Fermion Droplets," arXiv:hep-th/0604209.

[19] S. Yamaguchi, "Wilson loops of anti-symmetric representation and D5-branes," arXiv:hep-th/0603208.

[20] O. Aharony, J. Marsano, S. Minwalla, K. Papadodimas and M. Van Raamsdonk, "The Hagedorn / deconfinement phase transition in weakly coupled large N gauge theories," Adv. Theor. Math. Phys. 8 (2004) 603 [arXiv:hep-th/0310285].

[21] S. A. Hartnoll and S. Prem Kumar, "AdS black holes and thermal Yang-Mills correlators," JHEP 0512 (2005) 036 [arXiv:hep-th/0508092].

[22] S. A. Hartnoll and S. Prem Kumar, "Multiply wound Polyakov loops at strong coupling," arXiv:hep-th/0603190.

[23] L. Fidkowski and S. Shenker, "D-brane instability as a large N phase transition," arXiv:hep-th/0406086.

[24] A. Dymarsky, S. Gubser, Z. Guralnik and J. Maldacena, "Calibrated surfaces and supersymmetric Wilson loops," arXiv:hep-th/0604058.

[25] R. Dijkgraaf and C. Vafa, "Matrix models, topological strings, and supersymmetric gauge theories," Nucl. Phys. B 644, 3 (2002) [arXiv:hep-th/0206255]. 